\title{
Functional Performance as a Predictor of Injurious Falls in Older Adults
}

\author{
Rachel E. Ward, PhD, MPH, ${ }^{a, b, c}$ Suzanne G. Leveille, RN, PhD, ${ }^{d}$ Marla K. Beauchamp, PT, PhD, ${ }^{a, b}$ \\ Thomas Travison, PhD, ${ }^{e}$ Neil Alexander, MD, ${ }^{f, g}$ Alan M. Jette, PT, PhD, ${ }^{c}$ and Jonathan \\ F. Bean, $M D, M S, M P H^{a, b}$
}

OBJECTIVES: To determine whether a performance battery and its components aid in predicting injurious falls.

DESIGN: Longitudinal analysis; prospective cohort study. SETTING: Clinical site.

PARTICIPANTS: Boston-area community-dwelling adults $(\mathrm{N}=755$; mean age $\pm \mathrm{SD} 78.1 \pm 5.4,64.1 \%$ female, $77.6 \%$ white).

MEASUREMENTS: Baseline functional performance was determined according to the Short Physical Performance Battery (SPPB), measuring balance, gait speed, and five repeated chair stands. Fall history (past year) and efficacy in performing 10 daily activities without falling were assessed. Falls were assessed using a daily calendar over 4 years. Injurious falls were defined as resulting in fractures, sprains, dislocations, pulled or torn muscles, ligaments, or tendons or seeking medical attention.

RESULTS: Poorest chair stand performance $(\geq 16.7 \mathrm{sec}-$ onds) was associated with greater hazard of injurious falls than in all other chair stand performance groups (hazard ratio $(\mathrm{HR})=1.96,95 \%$ confidence interval $(\mathrm{CI})=1.18$ 3.26 for $\geq 16.7$ vs. $13.7-16.6$ seconds; $\mathrm{HR}=1.65,95 \%$ $\mathrm{CI}=1.07-2.55$ for $\geq 16.7$ vs. $11.2-13.6$ seconds, $\mathrm{HR}=$ $1.60,95 \% \mathrm{CI}=1.03-2.48$ for $\geq 16.7$ vs. $<11.2$ seconds). SPPB did not predict injurious falls. Fall history predicted injurious falls ( $\mathrm{HR}=1.82,95 \% \mathrm{CI}=1.39-2.39)$; falls efficacy did not. Fall history and a slow chair stand $(<16.7$ seconds) had a 2 -year cumulative incidence rate of an injurious fall of $46 \%(95 \% \mathrm{CI}=0.34-0.58)$, nearly the

From the a Spaulding Rehabilitation Hospital, Boston; ${ }^{b}$ Department of Physical Medicine and Rehabilitation, Harvard Medical School,

Cambridge; 'Health and Disability Research Institute, School of Health, Boston University; ${ }^{\mathrm{d} C o l l e g e}$ of Nursing and Health Sciences, University of Massachusetts Boston; ${ }^{\mathrm{e}}$ Institute for Aging Research, Hebrew SeniorLife, Boston, Massachusetts; ${ }^{\mathrm{f}}$ Division of Geriatric and Palliative Medicine, Department of Internal Medicine, University of Michigan; and ${ }^{\mathrm{g}}$ Geriatric Research, Education and Clinical Center, Veterans Affairs Ann Arbor Health Care System, Ann Arbor, Michigan.

Address correspondence to Rachel E. Ward, Spaulding Hospital Cambridge, 1575 Cambridge St., Cambridge, MA 02138. E-mail: reward@partners.org

DOI: $10.1111 /$ jgs. 13203 combined rate of a positive fall history $(0.29,95 \%$ $\mathrm{CI}=0.25-0.34)$ and a slow chair stand alone $(0.21,95 \%$ $\mathrm{CI}=0.13-0.30)$.

CONCLUSION: An easily administered chair stand test may be sufficient for evaluating performance as part of a risk stratification strategy for injurious falls. J Am Geriatr Soc 63:315-320, 2015.

Key words: falls; injury; aged; risk assessment

$\mathrm{F}$ all-related injuries in older adults are a major public health problem. Annually, $35 \%$ to $40 \%$ of communitydwelling adults aged 65 and older fall, with $10 \%$ of those who fall suffering serious injury. ${ }^{1}$ Fall-related injuries are a major source of mortality, morbidity, and disability and can lead to loss of independence. ${ }^{2,3}$ In 2000, $\$ 19.2$ billion was spent on fall-related injuries in the United States. ${ }^{4}$ This is expected to climb $\$ 32.4$ billion by $2020 .^{5}$

The Centers for Disease Control and Prevention has released an algorithm for Falls Risk Assessment and Interventions to aid in care planning and prevention. ${ }^{6}$ This algorithm recommends assessing fall history, falls self-efficacy (e.g., worrying about falling), and functional performance, which may inform clinical decisions on patient education, referral to exercise or prevention programs, or conduct of multifactorial risk assessments and interventions. This algorithm was created to assess overall falls risk, although serious fall-related injuries have more direct consequences for health, function, and healthcare expenditures.

The algorithm evaluates three functional performance domains associated with falls ${ }^{7}$ and fall-related injuries: ${ }^{2}$ gait, lower-extremity strength or chair stand performance, and balance. Various tests assess these domains, although there is limited evidence regarding which test is most predictive of injurious falls. Few studies have investigated how these tests perform in combination with other brief assessments such as a falls history or self-efficacy. ${ }^{8,9}$

The Short Physical Performance Battery (SPPB) captures each of these functional domains with established cutpoints 
predictive of disability and mortality in older adults. ${ }^{10,11}$ The SPPB is easily administered, requires little equipment, and can be completed in less than 10 minutes. Despite this, it has not been evaluated longitudinally as a predictor of fall-related injuries. Using the Centers for Disease Control and Prevention Falls Risk Assessment and Interventions algorithm as a guide, whether functional performance, combined with fall history and falls efficacy, predicts time to incident injurious falls was examined. It was hypothesized that, combined with fall history and falls efficacy, the SPPB and/or its components would predict injurious falls.

\section{METHODS}

The Maintenance of Balance, Independent Living, Intellect and Zest in the Elderly (MOBILIZE) Boston Study was designed to assess risk factors and mechanisms of falls in a cohort of 765 community-dwelling older adults living in the Boston area. ${ }^{12}$ Eligibility included age 70 and older, ability to walk 20 feet without the aid of another person, and intention to stay in the Boston area for 2 years or longer. ${ }^{13}$ Exclusions were moderate to severe cognitive impairment (Mini-Mental State Examination (MMSE) score $<18)$, severe visual or hearing deficits, and terminal illness. The analysis included $755(98.7 \%)$ participants. Participants with less than 90 days of follow-up because of withdrawal $(\mathrm{n}=8)$ from the study or death $(\mathrm{n}=2)$ were excluded from the analysis.

\section{Injurious Falls}

Falls were defined as unintentionally coming to rest on the ground or another lower level not resulting from a major health event (e.g., myocardial infarction) or an overwhelming external hazard (e.g., vehicular accident). ${ }^{14}$ Participants mailed daily falls calendars to the study site monthly. ${ }^{15}$ Falls were assessed during a maximum followup of 4.3 years. Associated injuries were ascertained through structured interviews. Injurious falls were defined according to fractures; sprains; dislocations; pulled or torn muscles, ligaments, or tendons; or by seeking medical attention. Falls data were obtained for $98.5 \%$ of follow-up months in the first year, $90.8 \%$ in the second year, $88.2 \%$ in the third year, and $81.2 \%$ in the fourth year. ${ }^{16}$

\section{The SPPB}

The SPPB is a well-established, reliable, valid measure of lower-extremity performance. ${ }^{10,11}$ It includes a test of standing balance, a timed 4-m usual-pace walk, and a timed test of five repeated chair stands. Each test is scored from 0 to 4 , with a maximum summed score of 12 for the three tests and higher scores indicating better functioning. Scores were categorized based on previously validated cutpoints (Tables 1 and 2). ${ }^{10}$ The SPPB is predictive of disability, hospitalization, and mortality in older populations. ${ }^{10,11}$

\section{Baseline Characteristics}

Baseline assessments included a home interview conducted by a trained research assistant, followed by a clinic assessment visit within approximately 2 weeks of the inter-
Table 1. Risk of Injurious Falls According to Short Physical Performance Battery (SPPB) Score, ${ }^{\mathrm{a}}$ Fall History, and Falls Efficacy

\begin{tabular}{|c|c|c|}
\hline & $\begin{array}{c}\text { Model 1: Without } \\
\text { SPPB } \\
\left(\text { AIC }=2,696.4^{\text {b }}\right)\end{array}$ & $\begin{array}{c}\text { Model 2: With } \\
\text { SPPB } \\
\left(\text { AIC }=2,699.1^{\text {b }}\right)\end{array}$ \\
\hline Predictor & \multicolumn{2}{|c|}{$\begin{array}{c}\text { Hazard Ratio (95\% Confidence } \\
\text { Interval) }\end{array}$} \\
\hline Fall history (yes vs no) & $1.82(1.39-2.39)^{\mathrm{C}}$ & $1.84(1.40-2.42)^{c}$ \\
\hline $\begin{array}{l}\text { Falls Efficacy Scale } \\
\text { (per standard deviation) }^{\text {d }}\end{array}$ & $0.90(0.80-1.02)$ & $0.92(0.81-1.04)$ \\
\hline \multicolumn{3}{|l|}{ SPPB score (1-12) } \\
\hline $1-3$ vs $4-6$ & - & $0.63(0.30-1.34)$ \\
\hline $1-3$ vs $7-9$ & - & $0.89(0.44-1.79)$ \\
\hline $1-3$ vs $10-12$ & - & $0.98(0.48-1.99)$ \\
\hline $4-6$ vs $7-9$ & - & $1.39(0.87-2.22)$ \\
\hline $4-6$ vs $10-12$ & - & $1.53(0.96-2.44)$ \\
\hline $7-9$ vs $10-12$ & - & $1.10(0.80-1.52)$ \\
\hline Age & $1.03(1.00-1.05)^{\mathrm{C}}$ & $1.02(1.00-1.05)$ \\
\hline Male sex & 0.77 (0.58-1.03) & $0.77(0.58-1.03)$ \\
\hline White race & $1.61(1.11-2.33)^{\mathrm{C}}$ & $1.61(1.11-2.33)^{\mathrm{C}}$ \\
\hline Psychoactive drug use & $1.76(1.11-2.80)^{\mathrm{C}}$ & $1.76(1.11-2.80)^{\mathrm{c}}$ \\
\hline Depression & $1.60(1.03-2.74)^{\mathrm{C}}$ & $1.60(1.03-2.47)^{\mathrm{C}}$ \\
\hline
\end{tabular}

$\mathrm{n}=32(4.3 \%)$ for SPPB $=1-3, \mathrm{n}=68(9.1 \%)$ for $\mathrm{SPPB}=4-6, \mathrm{n}=204$ $(27.3 \%)$ for SPPB $=7-9, \mathrm{n}=443(59.3 \%)$ for SPPB $=10-12$.

${ }^{\mathrm{a}}$ Higher scores indicate better physical functioning.

${ }^{\mathrm{b}}$ Lower values indicate better model performance.

${ }^{\mathrm{c}} P<.05$.

${ }^{\mathrm{d}}$ Falls Efficacy Scale range 1 (not at all confident) to 10 (extremely confident).

$\mathrm{AIC}=$ Akaike information criterion

view. Fall history (yes/no) within the past year was assessed according to self-report. Falls self-efficacy was measured using the Falls Efficacy Scale, ${ }^{17}$ for which participants were asked to rate their level of confidence from 1 (not at all confident) to 10 (extremely confident) in performing 10 daily activities without falling.

Covariates such as age, sex, race, body mass index (BMI), and baseline health conditions known and hypothesized to be related to falls and fall-related injuries were considered in the analysis. ${ }^{2,6}$ Psychotropic medication use (yes/no) included use of antidepressants, antipsychotics, hypnotics, benzodiazepines, and other sedatives. Cognitive impairment was defined as a score of less than 24 on the MMSE. ${ }^{13}$ Depression was assessed using a modified Center for Epidemiologic Studies Depression Scale. ${ }^{18,19}$ Vision was assessed using a light box at a 10 -foot distance $;^{20}$ visual deficit was defined as scoring in the lowest quartile. Orthostatic hypotension was defined as a reduction of systolic blood pressure of $20 \mathrm{mmHg}$ or more or of diastolic blood pressure of $10 \mathrm{mmHg}$ or more within 3 minutes of standing. ${ }^{21}$ Sensory impairment was defined as being unable to feel a 10-g Semmes-Weinstein monofilament on the dorsum of either great toe. ${ }^{22}$

\section{Statistical Analysis}

Multivariable Cox proportional hazards models were built to predict incident injurious falls. Covariates univariately related to injurious falls $(\alpha=0.1)$ were included in the models to prevent overfitting. The predictors fall history 
Table 2. Risk of Injurious Falls According to Short Physical Performance Battery (SPPB) Component Scores, Fall History, and Falls Efficacy

Model 1: Gait Speed ${ }^{\mathrm{a}}$

$\left(A I C=2,697.7^{b}\right)$
Model 2: Chair Stands ${ }^{c}$

$\left(A I C=2,309.5^{b}\right)$
Model 3: Balance

$\left(A I C=2,670.4^{b}\right)$

\section{Predictor}

Hazard Ratio (95\% Confidence Interval)

\begin{tabular}{llll}
\hline $\begin{array}{l}\text { Fall history (yes vs no) } \\
\text { Falls Efficacy Scale } \\
\text { per standard deviation) }^{f}\end{array}$ & $1.80(1.37-2.36)^{\mathrm{e}}$ & $1.79(1.33-2.40)^{\mathrm{e}}$ & $1.79(1.36-2.35)^{\mathrm{e}}$ \\
SPPB Component Scores $(1-4)^{\mathrm{g}}$ & $0.93(0.82-1.06)$ & $0.93(0.79-1.08)$ & $0.91(0.79-1.03)$ \\
$\quad 1$ vs & & & \\
1 vs 3 & $0.61(0.25-1.50)$ & $1.96(1.18-3.26)^{\mathrm{e}}$ & $1.54(0.87-2.75)$ \\
1 vs 4 & $0.85(0.35-2.06)$ & $1.65(1.07-2.55)^{\mathrm{e}}$ & $1.08(0.64-1.81)$ \\
2 vs 3 & $1.01(0.41-2.47)$ & $1.60(1.03-2.48)^{\mathrm{e}}$ & $1.35(0.82-2.22)$ \\
2 vs & $1.40(0.87-2.26)$ & $0.84(0.54-1.31)$ & $0.70(0.43-1.15)$ \\
3 vs 4 & $1.66(1.05-2.63)^{\mathrm{e}}$ & $0.81(0.52-1.27)$ & $0.88(0.56-1.39)$ \\
Age & $1.19(0.83-1.69)$ & $0.97(0.68-1.37)$ & $1.26(0.90-1.75)$ \\
Male & $1.02(1.00-1.05)$ & $1.03(1.01-1.06)^{\mathrm{e}}$ & $1.03(1.00-1.05)$ \\
White & $0.80(0.60-1.07)$ & $0.69(0.50-0.94)$ & $0.80(0.60-1.07)$ \\
Psychoactive drug use & $1.67(1.15-2.42)^{\mathrm{e}}$ & $1.59(1.07-2.38)^{\mathrm{e}}$ & $1.50(1.04-2.17)^{\mathrm{e}}$ \\
Depression & $1.75(1.10-2.79)^{\mathrm{e}}$ & $1.87(1.16-3.01)^{\mathrm{e}}$ & $1.66(1.04-2.64)^{\mathrm{e}}$ \\
\hline
\end{tabular}

${ }^{\mathrm{a}} 1=<0.46 \mathrm{~m} / \mathrm{s}(\mathrm{n}=21,2.8 \%) ; 2=0.46-0.64 \mathrm{~m} / \mathrm{s}(\mathrm{n}=70,9.4 \%) ; 3=0.65-0.82 \mathrm{~m} / \mathrm{s}(\mathrm{n}=140,18.7 \%) ;$ and $4=\geq 0.83(\mathrm{n}=516,69.1 \%)$.

${ }^{\mathrm{b}}$ Lower scores indicate better model performance.

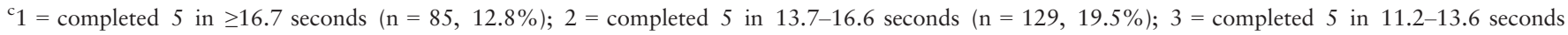
$(\mathrm{n}=232,32.7 \%)$, and $4=$ completed 5 in $<11.2$ seconds $(\mathrm{n}=217,32.7 \%)$.

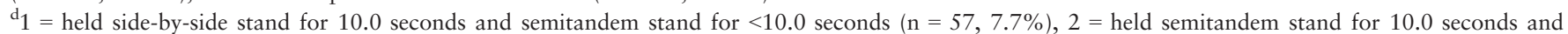

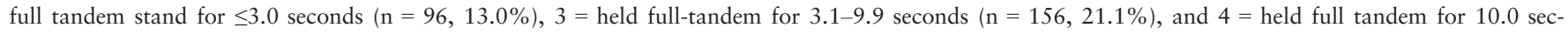
onds $(\mathrm{n}=430,58.2 \%)$.

${ }^{\mathrm{e}} P<.05$.

${ }^{\mathrm{f}}$ Range 1 (not at all confident) to 10 (extremely confident).

${ }^{\mathrm{g}}$ Higher scores indicate better physical function.

$\mathrm{AIC}=$ Akaike information criterion.

and falls efficacy score were adjusted for age, sex, race, psychotropic medication use, and depression, 2,6 and SPPB or SPPB component scores were added as ordinal predictors in separate models. The Akaike information criterion was used to determine goodness of fit. The proportional hazards assumption was checked by testing the relationship between injurious falls and the interaction between the predictors or covariates and the log of survival time $(\alpha=0.05)$. Cumulative incidence was calculated according to risk group and plotted against follow-up time. Two sensitivity analyses were performed, one assessing the relationships between continuous functional performance (gait speed and chair rise time) and injurious falls using Cox proportional hazards regression and one using classification and regression tree (CART) analysis in R statistical software (R Foundation for Statistical Computing, Vienna, Austria $)^{23}$ to determine the cutpoint or threshold of continuous performance most predictive of injurious falls. Analyses other than CART were performed using SAS version 9.3 (SAS Institute, Inc., Cary, NC).

\section{RESULTS}

The proportional hazards assumption was met. Participants had a median follow-up time of 2.43 years (interquartile range 1.40-3.23). Over the follow-up, 221 participants $(29 \%)$ experienced one or more injurious falls. The mean age of the 755 participants was $78.1 \pm 5.4$; $64.1 \%$ were female, and $77.6 \%$ were white.
Adjusting for covariates, fall history predicted incident injurious falls (hazard ratio $(\mathrm{HR})=1.82,95 \%$ confidence interval $(\mathrm{CI})=1.39-2.39)$, whereas falls efficacy and SPPB score did not (Table 1). Participants with the poorest chair stand performance ( $\geq 16.7$ seconds) had a greater hazard of injurious falls than all other groups $(\mathrm{HR}=1.96,95 \%$ $\mathrm{CI}=1.18-3.26$ for $\geq 16.7$ vs. $13.7-16.6$ seconds, $\mathrm{HR}=$ $1.65,95 \% \mathrm{CI}=1.07-2.55$ for $\geq 16.7$ vs. $11.2-13.6$ seconds, $\mathrm{HR}=1.60,95 \% \mathrm{CI}=1.03-2.48$ for $\geq 16.7$ vs. $<11.2$ seconds) (Table 2). Inability to complete the chair stand $(\mathrm{n}=64)$ was not associated with greater hazard of injurious fall $(\mathrm{HR}=1.07,95 \% \mathrm{CI}=0.66-1.73$ vs all other participants). The second-poorest-performing gait speed group had a greater hazard of injurious fall than the highest-performing group ( $\mathrm{HR}=1.66,95 \% \mathrm{CI}=1.05-2.63)$.

Because the worst-performing chair stand group had the highest hazard of injurious falls, and the chair stand model had the best goodness of fit, the interaction between chair stand performance and fall history, which was not significantly associated with injurious falls was tested. The cumulative incidence of an injurious falls when having a slow chair stand (score $=1 ; \geq 16.7$ seconds) and a fall history were assessed together and separately was also assessed (Figure 1). A positive fall history and slow chair stand were associated with a 2 -year cumulative incidence of $46 \%(95 \% \mathrm{CI}=0.34-0.58)$ for an injurious fall, compared with $12 \%(95 \% \mathrm{CI}=0.10-0.15)$ for neither risk factor, $29 \%(95 \% \mathrm{CI}=0.25-0.34)$ for a fall history alone, and $21 \%(95 \% \mathrm{CI}=0.13-0.30)$ for a slow chair 

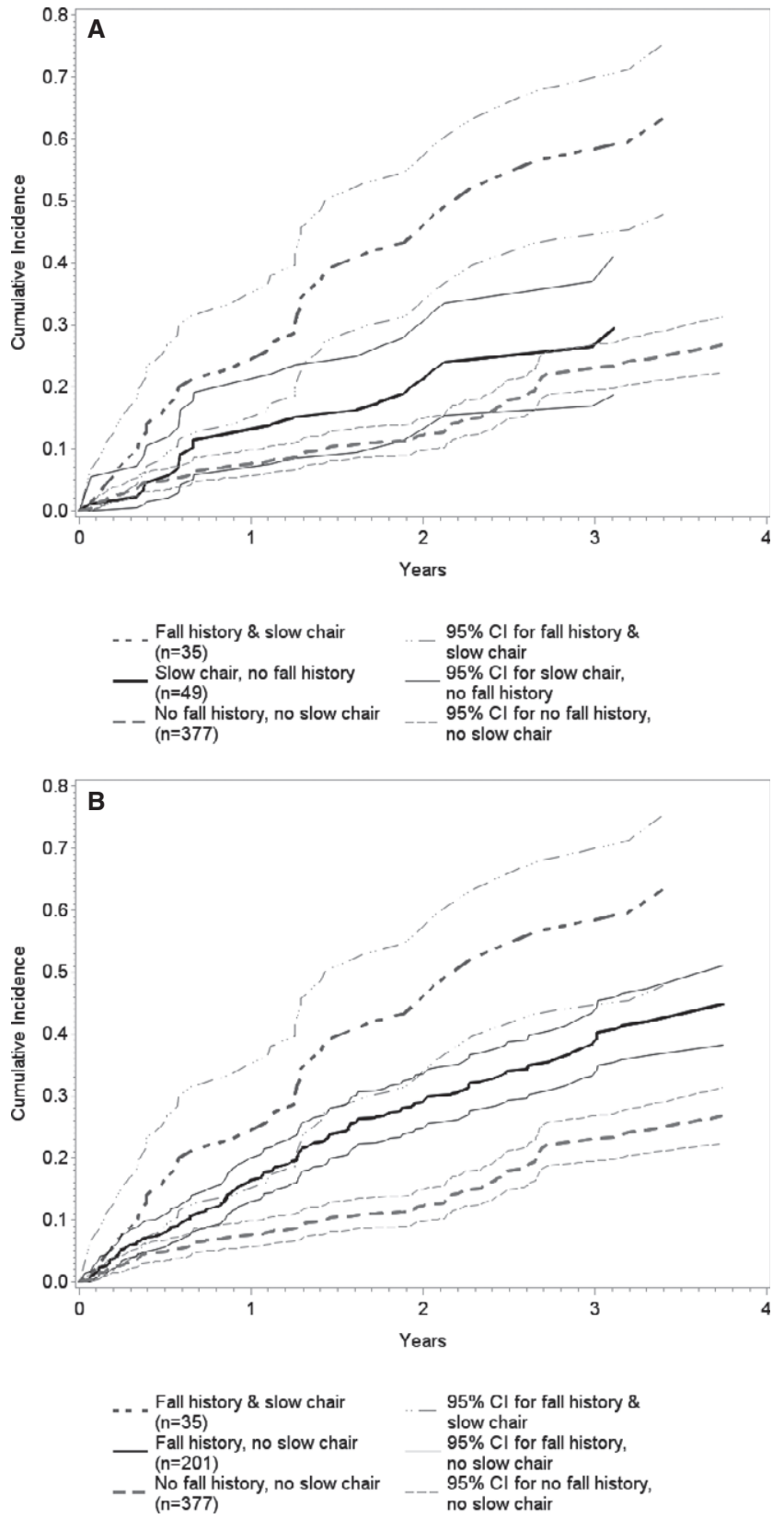

Figure 1. Cumulative incidence of injurious falls according to risk group. (A) Cumulative incidence rates of injurious falls across 4 years for the slow-chair-stand, no-fall-history risk group. (B) Cumulative incidence rates for the fallhistory, no-slow-chair-stand risk group. Both figures also show the cumulative incidence rates of the fall-history, slow-chair-stand risk group and the no-fall history, no-slowchair-stand risk group for comparison. Bold lines represent cumulative incident rates, and fine lines represent upper and lower bounds for $95 \%$ confidence intervals (CIs) of the cumulative incidence rates.

stand alone. Neither gait speed $(\mathrm{HR}=0.63,95 \%$ $\mathrm{CI}=0.33-1.20)$ nor chair rise time $(\mathrm{HR}=1.02,95 \%$ $\mathrm{CI}=0.99-1.02)$ was significant as a continuous predictor of injurious falls. Using CART, the most-predictive cutpoint of injurious falls in chair stand time was $15.5 \mathrm{sec}-$ onds or longer.

\section{DISCUSSION}

The utility of a lower-extremity function measure as part of an injurious falls risk assessment strategy in older adults was determined. Although SPPB score was not predictive of injurious falls in this cohort, a 16.7-second cutpoint for the five-repetition chair stand test was an independent predictor. Performing slower than this threshold and reporting one or more falls in the preceding year was associated with a compounded risk of injurious falls over 4 years. These findings inform fall risk assessment recommendations and stratification guidelines and extend them to include fall-related injuries.

Multiple components of functional performance were assessed separately and in combination using the SPPB to evaluate injurious falls risk. The chair stand component of the SPPB performed better than the combined score, which includes balance and gait speed. This has important clinical implications, suggesting that a short, simple chair stand test, which may identify lower-extremity weakness, poor muscle power, and limitations in dynamic balance, may be sufficient for evaluating performance as part of a risk stratification strategy for injurious falls.

Although the SPPB has not been assessed to the authors' knowledge as a predictor of injurious falls using prospective data, the chair stand test was retrospectively evaluated as a predictor of falls and fall-related fractures. ${ }^{24}$ The test was predictive of falls but not fall-related factures, although because 10 of 101 participants experienced fractures, lack of statistical power could account for this negative finding. In addition, previous studies have found that chair stand performance has been associated with recurrent falls, ${ }^{3,25,26}$ with one study reporting that chair stand performance was a better predictor of multiple falls than the one-leg standing balance test and the Timed Up and Go Test ${ }^{26}$ and another study reporting that it had predictive validity similar to that of the alternate-step test and the 6-m-walk test. ${ }^{25}$ None of these studies evaluated the association of these tests with injurious falls.

When the magnitude of the HRs for chair stand performance predicting injurious falls was examined, a HR closer to 1 was noted in the comparison between the poorest- and the best-performing group (1 vs 4 ) than in the comparison between the poorest- and the second-poorest-performing group (1 vs 2). Previous findings from this study show that individuals with high and low performance have higher rates of falls than those in the middle categories, ${ }^{27}$ suggesting that higher functioning may contribute to falls due to greater exposure to activities that may precipitate falls. It may be that individuals with poorer function are driving the significant relationship observed, whereas those with higher function are responsible for the unexpected trend in magnitude of the HRs. In addition, participants who were unable to complete the chair stand test did not have a greater hazard of fall-related injury, which was unexpected because it is likely that this group was the most impaired. One potential explanation is that this group's impairment may have decreased their mobility, which could have led to less exposure to situations in which falls might occur. Further investigation of fall-related injury risk is needed in these groups. 
Having a slow chair stand and a fall history together was not only associated with a greater incidence of injurious falls than having neither risk factor, but also resulted in nearly the combined risk of having either risk factor alone. This emphasizes the importance of assessing fall history and functional performance when estimating risk of fall-related injury. Although there was some overlap in the CIs for cumulative incidence in these risk groups, this is probably due to the small number of participants in the slow-chair-stand, fall-history risk group $(\mathrm{n}=35)$. Having only a positive fall history was also associated with a higher cumulative incidence of having an injurious fall than having neither risk factor. A slow chair stand without a positive fall history was associated with a marginally higher incidence and was not significantly different from that of the low-risk group, although this group may include older adults with poor function who may be at future risk of injurious falls and other unfavorable outcomes. This group also had large CIs, probably due to fewer participants $(\mathrm{n}=49)$.

Once individuals are stratified according to basic risk factors, those identified as high risk should undergo a more-comprehensive multifactorial risk assessment and treatment, if needed. ${ }^{6,28}$ Multifactorial assessments and treatment of individuals identified as being at high risk of falling can lead to a $30 \%$ to $40 \%$ reduction in falls. ${ }^{29}$ Consistent with this multifactorial approach, additional risk factors for fall-related injury were included in the analysis, and it was found that psychoactive medication use and depression were predictive, although they did not attenuate the effects of functional performance. More research is needed to evaluate whether multifactorial treatment strategies are similarly effective at preventing fallrelated injuries.

A major strength of this study is the collection of longitudinal falls data through monthly calendars over a maximum follow-up of 4.3 years. For functional performance, previously defined cutpoints were used that have been validated in large populations of older adults, which may make them more generalizable. ${ }^{10}$ Sensitivity analyses were performed to test whether continuous chair rise time and gait speed predicted injurious falls, which they did not, supporting the findings of a threshold effect with chair rise time. A CART analysis, which determines the most-predictive cutpoint from a continuous measure, was also performed, resulting in a similar cutpoint of 15.5 seconds in chair rise time. A potential future direction is to determine the predictive validity of each of these cutpoints in a separate study population.

Given its direct relationship with poor health and function and greater healthcare expenditures, the outcome of serious fall-related injury is particularly salient to older adults, the healthcare system, and the economy at large. Much of the literature focuses solely on falls, ignoring resulting injuries. Risk factors that are specific to or have greater importance for fall-related injuries are crucial to include in studies focusing on risk assessment and treatment.

Limitations of this study include that specific findings may be limited to community-dwelling older adults and may not be generalizable to other populations, such as institutionalized or frail older adults or those with significant cognitive impairments. Because of the small number of participants in the slow-chair-stand, fall-history group and the slow-chair-stand, no-fall-history group, there may have been insufficient power to fully detect differences in cumulative incidence of injurious falls between these and other groups. In addition, injurious falls risk was assessed only at baseline, and individuals' functional status can fluctuate over time, but the baseline assessment may help shed light on what can be predicted from a onetime medical assessment anywhere from 0 to 4 years later, as Figure 1 illustrates. Finally, the analysis was designed to assess time to first injurious fall. An important future direction is to assess whether the SPPB or its components can stratify individuals at risk for multiple future injurious falls.

\section{CONCLUSION}

The chair stand assessment predicts injurious falls and may have clinical utility when implementing a risk assessment and treatment strategies. This assessment is easy and quick to use in a busy clinical practice. These findings support the use of current algorithms including measures of fall history and functional performance and extend the use of these tools to risk estimation of fall-related injury. Estimation of injurious falls risk has particularly important implications for prevention of disability and mortality in older adults ${ }^{30}$ and reducing healthcare use and expenditures. ${ }^{4}$ Future studies should investigate the effectiveness of intervening on groups stratified according to injurious falls risk and how treatment strategies should differ based on results from risk assessment.

\section{ACKNOWLEDGMENTS}

An abstract reporting results from these data was accepted as a poster presentation at the International Society for Posture and Gait Research World Congress, Vancouver, Canada, June 30, 2014.

This work was supported by the National Institute on Aging Research Nursing Home Program Project P01AG004390 and Research Grants R01-AG026316 and R37-AG25037.

Conflict of Interest: Alan M. Jette, Jonathan F. Bean, and Suzanne G. Leveille declare grant support from National Institute on Aging Grant 5 R01-AG032052-03 and from the National Center for Research Resources in Grant 1 UL1-RR025758-01 to the Harvard Clinical and Translational Science Center. Rachel E. Ward is supported by National Institute on Disability and Rehabilitation Research Grant H133P120001. Marla K. Beauchamp is supported by a fellowship from the Canadian Institutes of Health Research.

Author Contributions: Leveille, Bean, and Jette: study concept and design, data acquisition and interpretation, preparation of manuscript. Ward, Travison: data analysis and interpretation, preparation of manuscript. Beauchamp, Alexander: data interpretation, preparation of manuscript.

Sponsor's Role: The funding institutes had no role in the design, methods, subject recruitment, data collection, analysis, or preparation of manuscript or in the decision to submit the manuscript for publication. 


\section{REFERENCES}

1. Rubenstein LZ, Josephson KR. The epidemiology of falls and syncope. Clin Geriatr Med 2002;18:141-158.

2. Tinetti ME, Doucette J, Claus E et al. Risk factors for serious injury during falls by older persons in the community. J Am Geriatr Soc 1995;43:12141221.

3. Nevitt MC, Cummings SR, Kidd S et al. Risk factors for recurrent nonsyncopal falls. A prospective study. JAMA 1989;261:2663-2668.

4. Stevens JA, Corso PS, Finkelstein EA et al. The costs of fatal and non-fatal falls among older adults. Inj Prev 2006;12:290-295.

5. Englander F, Hodson TJ, Terregrossa RA. Economic dimensions of slip and fall injuries. J Forensic Sci 1996;41:733-746.

6. Algorithm for Fall Risk Assessment and Interventions. STEADI Tool Kit for Health Care Providers 2012 [on-line]. Available at http:/www.cdc.gov/ homeandrecreationalsafety/pdf/steadi/algorithm_fall_risk_assessment.pdf Accessed April 14, 2014.

7. Ganz DA, Bao Y, Shekelle PG et al. Will my patient fall? JAMA 2007;297:77-86.

8. Lamb SE, McCabe C, Becker C et al. The optimal sequence and selection of screening test items to predict fall risk in older disabled women: The Women's Health and Aging Study. J Gerontol A Biol Sci Med Sci 2008;63A:1082-1088.

9. Nandy S, Parsons S, Cryer C et al. Development and preliminary examination of the predictive validity of the Falls Risk Assessment Tool (FRAT) for use in primary care. J Public Health (Oxf) 2004;26:138-143.

10. Guralnik JM, Simonsick EM, Ferrucci L et al. A short physical performance battery assessing lower extremity function: Association with selfreported disability and prediction of mortality and nursing home admission. J Gerontol 1994;49:M85-M94.

11. Guralnik JM, Ferrucci L, Pieper CF et al. Lower extremity function and subsequent disability: Consistency across studies, predictive models, and value of gait speed alone compared with the short physical performance battery. J Gerontol A Biol Sci Med Sci 2000;55A:M221-M231.

12. Leveille SG, Kiel DP, Jones RN et al. The MOBILIZE Boston Study: Design and methods of a prospective cohort study of novel risk factors for falls in an older population. BMC Geriatr 2008;8:16.

13. Folstein MF, Folstein SE, McHugh PR. 'Mini-mental state'. A practical method for grading the cognitive state of patients for the clinician. J Psychiatr Res 1975;12:189-198.

14. The prevention of falls in later life. A report of the Kellogg International Work Group on the Prevention of Falls by the Elderly. Danish Med Bull 1987;34(Suppl 4):1-24.

15. Tinetti ME, Liu WL, Claus EB. Predictors and prognosis of inability to get up after falls among elderly persons. JAMA 1993;269:65-70.
16. Kelsey JL, Procter-Gray E, Hannan MT et al. Heterogeneity of falls among older adults: Implications for public health prevention. Am J Public Health 2012;102:2149-2156.

17. Tinetti ME, Richman D, Powell L. Falls efficacy as a measure of fear of falling. J Gerontol 1990;45:P239-P243.

18. Eaton W, Muntaner C, Smith C et al. Center for Epidemiologic Studies Depression Scale: Review and Revision (CESD and CESD-R). In: Maruish M, ed. The Use of Psychological Testing for Treatment Planning and Outcomes Assessment, Vol. 3. Mahwah, NJ: Lawrence Erlbaum Assoc Inc., 2004, pp 363-377.

19. Roberts RE, Vernon SW. The Center for Epidemiologic Studies Depression Scale: Its use in a community sample. Am J Psychiatry 1983;140:41-46.

20. Wood KM, Edwards JD, Clay OJ et al. Sensory and cognitive factors influencing functional ability in older adults. Gerontology 2005;51:131-141.

21. The Consensus Committee of the American Autonomic Society and the American Academy of Neurology. Consensus statement on the definition of orthostatic hypotension, pure autonomic failure, and multiple system atrophy. Neurology 1996;46:1470.

22. Perkins BA, Olaleye D, Zinman B et al. Simple screening tests for peripheral neuropathy in the diabetes clinic. Diabetes Care 2001;24:250 256.

23. Kabacoff RI. Tree-Based Models. Quick-R 2014 [on-line]. Available at http://www.statmethods.net/advstats/cart.html Accessed July 8, 2014.

24. Zhang F, Ferrucci L, Culham E et al. Performance on five times sit-to-stand task as a predictor of subsequent falls and disability in older persons. J Aging Health 2013;25:478-492.

25. Tiedemann A, Shimada H, Sherrington C et al. The comparative ability of eight functional mobility tests for predicting falls in community-dwelling older people. Age Ageing 2008;37:430-435.

26. Buatois S, Miljkovic D, Manckoundia P et al. Five times sit to stand test is a predictor of recurrent falls in healthy community-living subjects aged 65 and older. J Am Geriatr Soc 2008;56:1575-1577.

27. Quach L, Galica AM, Jones RN et al. The nonlinear relationship between gait speed and falls: The Maintenance of Balance, Independent Living, Intellect, and Zest in the Elderly of Boston Study. J Am Geriatr Soc 2011;59:1069-1073.

28. Sherrington C, Tiedemann A, Fairhall $\mathrm{N}$ et al. Exercise to prevent falls in older adults: An updated meta-analysis and best practice recommendations. N S W Public Health Bull 2011;22:78-83.

29. Chang JT, Morton SC, Rubenstein LZ et al. Interventions for the prevention of falls in older adults: Systematic review and meta-analysis of randomised clinical trials. BMJ 2004;328:680.

30. Magaziner J, Simonsick EM, Kashner TM et al. Predictors of functional recovery one year following hospital discharge for hip fracture: A prospective study. J Gerontol 1990;45:M101-M107. 